\title{
Control of Active Turbulence through Addressable Soft Interfaces
}

\author{
P. Guillamat, ${ }^{1,2, a)}$ J. Hardoüin, ${ }^{1,2}$ B. M. Prat, ${ }^{1,2}$ J. Ignés-Mullol, ${ }^{1,2, b)}$ and F. Sagués ${ }^{1,2}$ \\ 1) Department of Materials Science and Physical Chemistry, Universitat de Barcelona \\ ${ }^{2)}$ Institute of Nanoscience and Nanotechnology, IN2UB, Universitat de Barcelona
}

(Dated: 9 November 2017)

We present an experimental study of a kinesin/tubulin active nematic formed at different oil interfaces. By tuning the interfacial rheology of the contacting oil, we have been able to condition and control the seemingly chaotic motion that characterizes the self-sustained active flows in our preparations. The active nematic is inherently unstable and spontaneously develops defects from an initial homogeneous state. We show that the steady state and, in particular, the density and dynamics of the defects strongly depends on the rheology of the contacting oil. Using a Smectic-A thermotropic liquid crystal as the oil phase, we pattern the interface thanks to the anisotropy of the shear viscosity in this material. The geometry of the active nematic adapts to the boundary conditions at the interface by changing from the so-called active turbulent regime to laminar flows along the easy flow directions. The latter can be either a lattice of self-assembled circular paths or reconfigurable homogeneous orientations that can be addressed by means of an external magnetic field. We show that, under all confinement conditions, the spatiotemporal modes exhibited by the active liquid are consistent with a single intrinsic length scale, which can be tuned by the material parameters, and obey basic topological requirements imposed on the defects that drive the active flows. Future control strategies, including a tunable depleting agent, are discussed.

PACS numbers: 87.16.Ka,61.30.Jf

Keywords: soft active matter, active turbulence, active nematic, smectic liquid crystals

\section{INTRODUCTION}

Active matter refers to systems constituted by selfdriven units, which exert forces to their surroundings and move autonomously by consuming stored or ambient free energy. In high density regimes, the action of the active units becomes dominated by the interaction with their neighbours, often leading to the assumption of average common directions, and to complex collective behaviours $^{1,2}$. Most examples of active matter come from living systems, and span a wide range of length scales, from flocks of birds or herds of sheep to the intracellular matrix. Because of the involved length and time scales and because of its dynamically self-assembled nature, the study of the cell cytoskeleton has arisen enormous interest among Soft Condensed Matter physicists. So far, however, most of the research has a theoretical standpoint due to the experimental complexity of living entities. Recently, experimental realisations have increased in part thanks to the successful in-vitro reconstitutions involving a minimal number of active biological ingredients. This has resulted in out-of-equilibrium materials that are generally referred to as active soft matter, or active liquids ${ }^{3-5}$. These materials, which contain filamentous cytoskeleton proteins and complementary molecular motors (proteins that are responsible of active intracellular transport at the expense of ATP (adenosine triphosphate) hydrolysis), are model systems to understand in-

\footnotetext{
a) Current address: Biochemistry Department, University of Geneva.

b) Electronic mail: jignes@ub.edu.
}

tracellular processes with the potential to develop novel biomaterials. A particularly remarkable example is the so-called active nematic, obtained by the condensation at a water/oil interface of a gel of microtubules that are actively cross-linked by clusters of the complementary motor kinesin ${ }^{5}$. In spite of the emergence of collective spatiotemporal phenomena and long-range orientational order, the long time evolution of these systems is unpredictable. The spatial arrangements and dynamics of these protein suspensions cannot be commanded by external electric or magnetic fields, commonly employed in classical Soft Matter systems, such as colloidal suspensions of thermotropic liquid crystals, thus preventing the full potential of active biomimetic materials from being unleashed.

Another interesting common feature between some active liquids and passive Soft Matter ${ }^{6}$ is the influence of bulk or interfacial confinement ${ }^{7-12}$. For instance, control of the anchoring conditions on confining surfaces is a well-known and essential tool in liquid crystal research and technology. This influence of the physicochemical interactions with a substrate is also ubiquitous in active biosystems. For instance, cells in some tissues adapt the rheology of their cytoskeleton to the stiffness of their supporting substrate ${ }^{13}$, and epithelial cells evolve differently according to the substrate stiffness ${ }^{14}$, a phenomenon known as durotaxis. In a cell biology context, this is also an important issue in relation to cytoplasmic streaming in eucaryotes ${ }^{15}$. Remarkably, a number of common diseases are directly or indirectly associated with a faulty cellular response to the mechanical properties of their surroundings ${ }^{16}$. Moreover, recent experiments with nematic liquid crystals under electrohydrodynamic instabilities $^{17}$ or with active biofluids ${ }^{18,19}$ have 
revealed that strong coupling with substrates can lead to the pattering of the otherwise chaotic flows. These results suggest that interaction with substrates may be a useful handle to control active fluids with techniques that are familiar to Soft Condensed Matter scientists and that can be easily implemented in a Soft Matter laboratory.

One of the hallmarks of active fluids is the onset of large-scale chaotic flows, with a reverse energy cascade originating at microscopic length scales by the dissipative active units and propagating towards larger length scales in the form of mesoscale vortices of different sizes. This self-sustained state has been called active or mesoscale turbulence, mostly because of its visual resemblance to classical inertial turbulence ${ }^{20-25}$. The in-depth analysis of active turbulence is still pending, although it has become clear that qualitative resemblance between both types of turbulence is only possible under confinement or at small scales ${ }^{20}$. In fact, a recent work based on numerical simulations has shown that both traditional and mesoscale turbulence follow the directed percolation universality class when constrained to a channel flow ${ }^{26}$.

There remain many open questions in the study of active turbulence, such as the primary driver of the turbulent flow, the nature of the primary excitations, which features are universal, etc ${ }^{24}$. A study of these issues, which involves an in-depth characterisation of the flowdynamics is beyond the scope of the current work. Instead, here we will provide a minimal geometrical characterisation of the steady-state chaotic regime in a tubulinkinesin active fluid, and show how this regime can be somewhat "tamed" by contact with patterned fluid interfaces.

In the next section, we describe the experimental system used to prepare and characterize the studied active nematic fluid. Next, we describe the primordial instability leading to the development of the steady state active turbulent regime, which we characterise and note its sensitivity to the interfacial rheology of the water/oil interface where it resides. Moreover, we describe the mechanisms with which the employed active nematic (AN) adapts to lateral confinement that is exerted by a Smectic-A (SmA) liquid crystal. The strong hydrodynamic coupling between the active and the passive fluids $^{27}$, along with the enormous anisotropy of the SmA interface, results in the rearrangement of the spontaneous disordered flows of the active material ${ }^{28}$. Moreover, the fact that the passive SmA can be easily oriented by an external magnetic field allows to externally command the AN, whose spontaneous flow is constrained along arbitrary easy-flow directions ${ }^{29}$. The ability to reversibly transform the geometry of the AN allows to characterize intrinsic length and time scales, to test the validity of existing scaling predictions, and to demonstrate an original mechanism for active fluids to adapt to geometrical confinement by redefining the role, rather than the value, of intrinsic length scales. Possible control strategies of the AN based on the tuning of its constituents are also discussed.
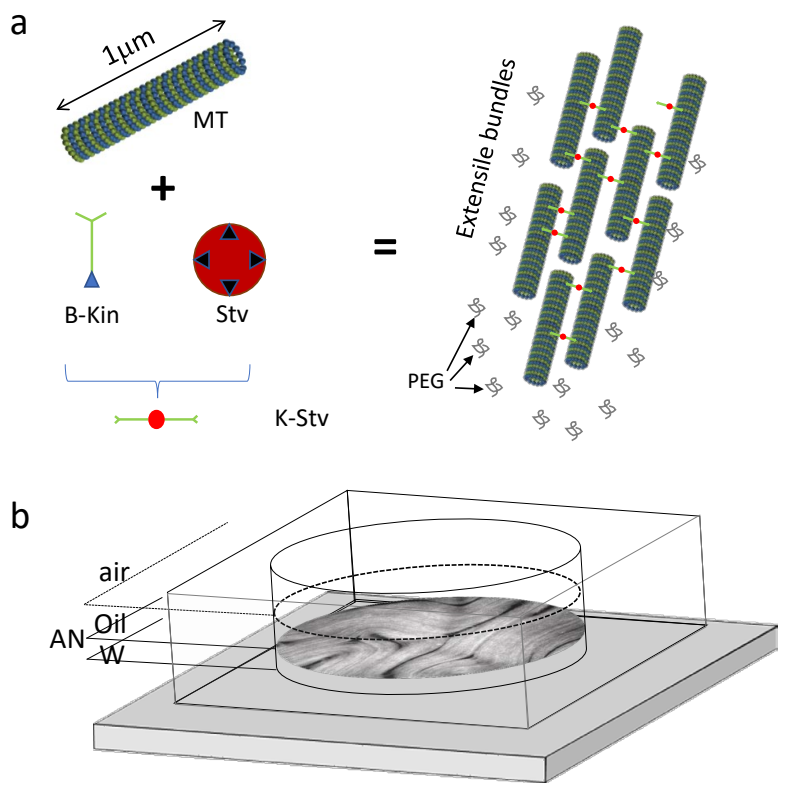

FIG. 1. (a) Fluorescently tagged microtubules (MT) from polymerized tubulin are brought together by the depleting action of PEG, and are crosslinked by clusters of Biotinylated Kinesin (B-Kin) and Streptavidin (Stv), resulting in active extensile bundles in an aqueous suspension. (b) A custom made cylindrical cell, $5 \mathrm{~mm}$ deep, $8 \mathrm{~mm}$ in diameter, is glued to a bioinert hydrophilic support glass plate. The active nematic self-assembles at the water/oil interface.

\section{EXPERIMENTAL METHODS}

\section{A. Materials}

Microtubules, $1-2 \mu \mathrm{m}$ in length, were polymerized from heterodimeric $(\alpha, \beta)$-tubulin (from bovine brain, obtained from the Brandeis University Biological Materials Facility), as previously described ${ }^{29}$. For the characterization with fluorescence microscopy, part of the initial tubulin (3\%) was fluorescently labelled. Biotinylated Drosophila Melanogaster heavy chain kinesin-1 K401-BCCP-6His was expressed in Escherichia Coli ${ }^{29}$. Dimeric motor clusters were obtained by incubating the kinesin motors with tetrameric streptavidin at a ratio 2:1. The active gel (Fig. 1.a) was obtained by mixing the microtubules with the kinesin clusters, which act as cross-linkers. The aqueous dispersion contains the non-adsorsbing polymer polyethylene glycol, (PEG, $20 \mathrm{kDa}$ ) that acts as a depleting agent, condensing the microtubules and kinesin clusters into thick elongated bundles. The presence of ATP together with an ATP-regenerating enzimatic system leads to the spontaneous elongation of the bundles. The triblock copolymer surfactant Pluronic F-127 was added at $1 \%(\mathrm{w} / \mathrm{w})$ to the aqueous phase in order to procure a biocompatible water/oil interface in subsequent steps.

The oily phase was constituted either of isotropic silicone oils of different viscosity (BlueSil ${ }^{\circledR}$, BlueStar 
Silicones), heptadecane (Sigma-Aldrich), or 4-cyano-4octylbiphenil (8CB, Synthon). The latter is a thermotropic liquid crystal between 21.4 and $40.4^{\circ} \mathrm{C}$, featuring a lamellar Smectic-A phase in the temperature range $21.4^{\circ} \mathrm{C}<T<33.4^{\circ} \mathrm{C}$.

\section{B. Methods}

The active nematic/passive liquid crystal interface was prepared in a cylindrical pool of diameter $5 \mathrm{~mm}$ and depth $8 \mathrm{~mm}$, manufactured with a block of polydimethylsiloxane (PDMS) using a custom mold. The block was glued onto a bioinert and superhydrophilic polyacrylamide coated glass (Fig. 1.b). Samples were placed inside a thermostatic oven in order to control the phase state of the oil phase. Notice that the active material is viable in a temperature range from $15^{\circ} \mathrm{C}$ to $40^{\circ} \mathrm{C}$, approximately. The ensemble could be placed in the cavity of a cylindrical permanent magnet array that provided a uniform magnetic field of up to $4 \mathrm{kG}$ parallel to the substrate ${ }^{29}$. This open-pool arrangement allowed to use high viscosity oils and also to mechanically perturb the interface to prepare an aligned initial state.

Routine observations of the active nematic were performed by means of conventional epifluorescence microscopy. Image acquisition was performed with a QImaging ExiBlue CCD cooled camera operated with ImageJ $\mu$-Manager open-source software. For sharper imaging of the interfacial region, we used laser-scanning confocal microscopy with a Leica TCS SP2 equipped with a photomultiplier as detector and a HeNe-633-nm laser as light source. In particular, sequences of confocal reflection images were acquired to perform image velocimetry using custom Matlab tools.

\section{RESULTS AND DISCUSSION}

\section{A. Hydrodynamic coupling across the interface}

Our experiments were performed with an active liquid constituted by fluorescently-labeled, micron-sized microtubules (MTs), bundled together by the depleting action of polyethylene glycol (PEG), and cross-linked by clusters of biotinylated kinesin motor proteins (Fig. 1.a). Under continuous supply of ATP, the tens-of-micronslong filamentous bundles are subjected to permanent internal stresses due to the action of the motor complexes. This aqueous preparation spontaneously self-assembles into a flow-permeated active gel ${ }^{30-32}$ that condenses as an AN layer at PEGylated surfactant-decorated oily interfaces. As a reference experiment, a layer of AN was interfaced with an isotropic oil in a custom-built cell (see Fig. 1.b). The AN textures that form upon continuous stretching and folding of MT bundles are punctured by disclinations that proliferate under high activity conditions, giving rise to a two-dimensional active turbulent

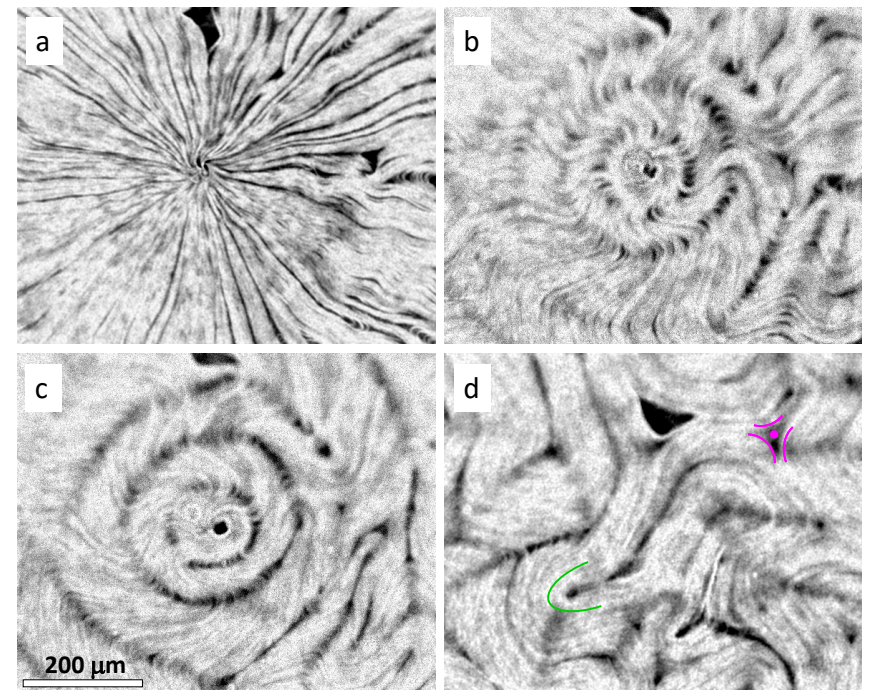

FIG. 2. (a)-(d) Fluorescence micrographs illustrating the sequence of instabilities leading to the formation of the active turbulent regime from the unstable aligned state (a). Elapsed times are $13 \mathrm{~s} \mathrm{(b),} 26 \mathrm{~s} \mathrm{(c),} \mathrm{and} 72 \mathrm{~s}$ (d). A parabolic $+1 / 2$ defect (green) and a hyperbolic $-1 / 2$ defect (purple) are highlighted in (d).

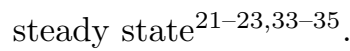

The onset of this steady state can be studied by preparing an initial condition where the MT bundles are aligned. In Fig. 2, this was achieved by applying a radial suction with a glass capillary taking advantage of our open cell setup. Once the flow was removed, the radially oriented extensile bundles developed a buckling instability perpendicular to the alignment direction (Fig. 2.b), with a well-defined length scale that could be directly assessed by observing the concentric anuli where the defect cores, devoid of fluorescent MTs, organized (Fig. 2.c). The azimuthal-aligned bundles were again unstable, this time with the formation of radially-moving defects. This hierarchy of defect proliferation soon led to a steady state regime (Fig. 2.d), where defect creation and annihilation balance, and the density of $+1 / 2$ and $-1 / 2$ defects remains constant ${ }^{22,27}$.

The fact that the AN layer evolves at the water/oil interface results in the active flows to propagate into the bulk fluids. Conversely, the viscosity of the contacting oil has a strong impact on the morphology and dynamics of the active nematic. In Fig. 3, we show snapshots of the AN in contact with oils of different viscosity, for the same activity (i.e., concentration of ATP). At higher oil viscosities, the speed of the AN defects and flow decrease, leading to an increase of the number of defects, which degrade the orientational order of the filamentous material. At the same time, the speed of the AN flow decreases. We also observed that textures in contact with oils of smaller viscosity appeared less fluorescent and more tenuous. This indicates that a large oil viscosity leads to thicker MT bundles while also amplifying the typical size 

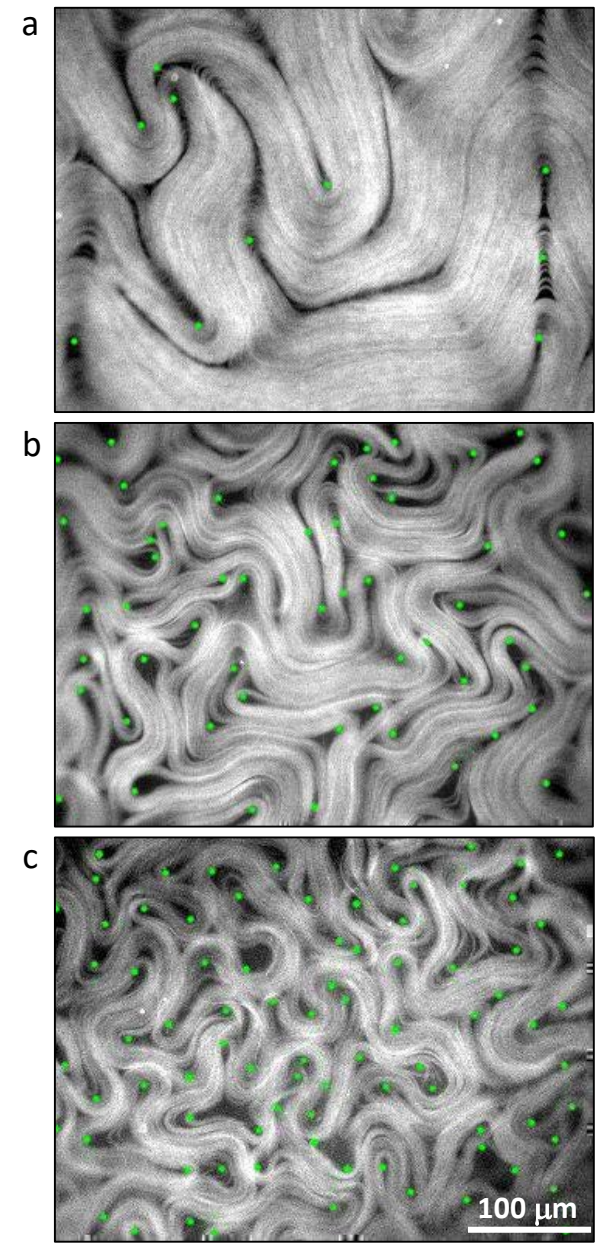

FIG. 3. (a)-(c) Fluorescence confocal micrographs of the active nematic in contact with silicone oils of viscosities 0.05 (a), 5 (b), and $300 \mathrm{~Pa} \mathrm{~s}$ (c). The focus of all $+1 / 2$ defects are marked by a green dot in (a)-(c).

of the empty regions that are the cores of the defect textures. In a recent work, we have employed this strong coupling to estimate the viscosity of the AN from measurements of active flow velocity using a model for the hydrodynamic coupling between the $\mathrm{AN}$ and the bulk fluid layers. ${ }^{27}$.

The observed strong coupling between the AN and the contacting bulk fluids, and the ability of the active liquid to adapt to oils of viscosities that span more than six orders of magnitude, suggests the possibility to change the spatial arrangement of the AN by interfacing it with an anisotropic liquid, i.e., a thermotropic liquid crystal, with well-defined easy flow directions. The reference state was the unconstrained active turbulent regime, when the AN evolves in contact with an isotropic oil. The geometry of this configuration is characterized in the following section.
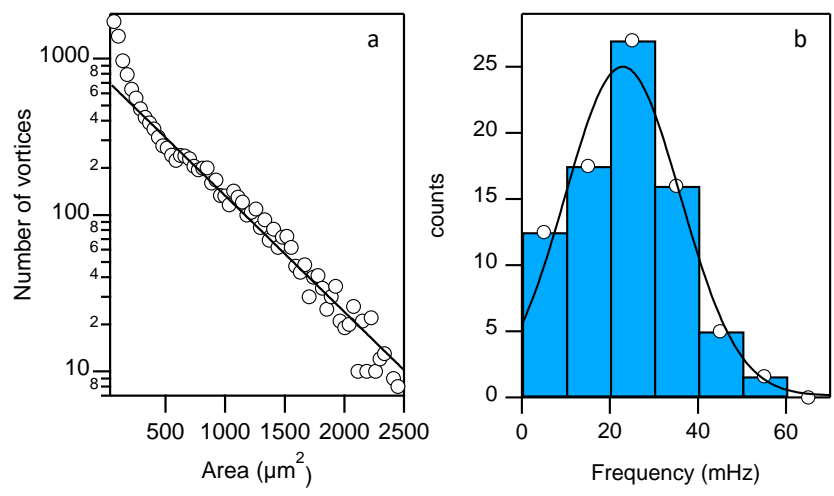

FIG. 4. (a) Distribution of vortex sizes in the active turbulent regime for the $\mathrm{AN}$ in contact with an isotropic oil of viscosity $12.5 \mathrm{~Pa}$ s. Experimental conditions of the $\mathrm{AN}$ are $[\mathrm{ATP}]=700 \mu \mathrm{M},[\mathrm{PEG}]=0.8 \% \mathrm{w} / \mathrm{w}$. The solid line is an exponential fit yielding a characteristic decay area of $585 \pm 17 \mu \mathrm{m}^{2}$. (b) Distribution of the inverse vortex lifetime (switching frequency) for the same regime and experimental conditions. The solid line is a Gaussian fit to the distribution yielding an average intrinsic frequency of $25 \pm 5 \mathrm{mHz}$.

\section{B. The reference active turbulence regime}

Active turbulence is a transversal concept that has been applied to characterize systems ranging from bacterial baths ${ }^{20,36}$ to in vitro aqueous reconstitutions of cytoskeletal proteins ${ }^{3-5}$. Different from classical inertiabased turbulence in Newtonian fluids, active turbulence is dominated by dissipation. Both types of turbulence, however, share the disparity of spatial scales and directions of motions in the abundant jets and swirls that characterize turbulent flows. In a recent analysis using computer simulations, Giomi characterized the geometry of the active turbulence regime of an $\mathrm{AN}$ in terms of the distribution of vortex sizes, and found the emergence of an intrinsic length scale in an exponential distribution ${ }^{22}$. In order to perform such analysis, a crucial issue is the definition of the size of a vortex. One possibility could be mapping the vorticity of the active flow field, associating each vortex to a region where vorticity has the same sign. However, one finds that a more consistent result is obtained by using the Okubo-Weiss parameter, $O W=\left(\partial_{x} v_{x}\right)^{2}+\partial_{y} v_{x} \cdot \partial_{x} v_{y}$, which provides with a standard criterion for vortex location, often used in fluid dynamics. We considered the extension of each vortex to be bound by the condition $O W<0^{22,37}$. Physically, this inequality defines a region, which includes the center of the vortex, within which any two advected tracer particles will remain at a finite distance from each other at all times. Conversely, advected particles in the region $O W>0$ will depart exponentially from each other. Using this criterion, we performed a statistical analysis of the vortex size distribution during the steady state active flow regime employing the local velocity of the AN obtained using image velocimetry analysis. In the data 
shown in Fig. 4.a, the AN was flowing in contact with an isotropic oil of viscosity $12.5 \mathrm{~Pa} \mathrm{~s}$, which is similar to the average viscosity of the liquid crystal employed below, so that results can be discussed together.

Although the range of vortex sizes within a given experiment spans less than a decade, there was a clear exponential trend in the distribution, which allowed to fit a characteristic vortex size, $A^{*}=585 \pm 17 \mu \mathrm{m}^{2}$ in these particular experimental conditions. This result is consistent with a characteristic length scale for the active turbulent regime, $l_{\alpha} \sim \sqrt{A^{*}}$. Numerical studies ${ }^{22}$ had revealed the existence of this intrinsic active length scale, which arises from the balance between active and passive stresses, and was predicted to exhibit the scaling relation $l_{\alpha} \sim \sqrt{K / \alpha}$, where $K$ is the bending rigidity of the material, and $\alpha$ is the active stress parameter, or activity. This length scale determines, for instance, the average defect distance in the steady state configuration (Fig. 2). Similarly, we could put into evidence an intrinsic time scale for this system by analyzing the typical lifetime of vortices. To obtain a statistical assessment of this magnitude, we divided the field of view in $30 \times 30 \mu \mathrm{m}^{2}$ cells, defining areas that can fit the characteristic vortex size found above. We then measured the area fraction with $O W<0$ for each of the cells as a function of time (during 1000s at an image sampling rate of $1 \mathrm{~Hz}$ ), and we computed the Fast Fourier Transform (FFT) of this signal. This analysis revealed a narrow distribution of frequencies, yielding a mean value $25 \pm 5 \mathrm{mHz}$ (Fig. 4.b), which results in $t^{*} \sim 40$ s for the vortex lifetime.

This unconstrained active turbulence regime, where the AN evolves in contact with an isotropic oil, was our reference state. Below we show how its geometry changes when the oil is replaced by an anisotropic fluid, either when the latter adopts its spontaneous configuration (Sec. III C) or when it is commanded by an external magnetic field (Sec. III D).

\section{Constrained active turbulence}

In order to constrain the AN to evolve along easy flow directions, one may resort to force the active material through engineered channels whose surface has been patterned using the techniques that have become standard in microfluidics. However, it turns out that the AN will only form when the extensile bundles are depleted towards a liquid interface, even if the latter is just a lubricating layer. In contact with a solid, only the bulk active gel is observed. A dramatic assessment of this result is seen in Fig. 5, where the active liquid was prepared by using the hydrocarbon oil heptadecane instead of the usual silicone oils. Heptadecane melts around $22^{\circ} \mathrm{C}$, so we could prepare the AN in contact with the liquid oil in the normal way, observing the formation of the active turbulent regime (Fig. 5a). Then, we lowered the temperature until the oil froze. Very quickly the AN vanished, leading to the disorganization of the bundles into a
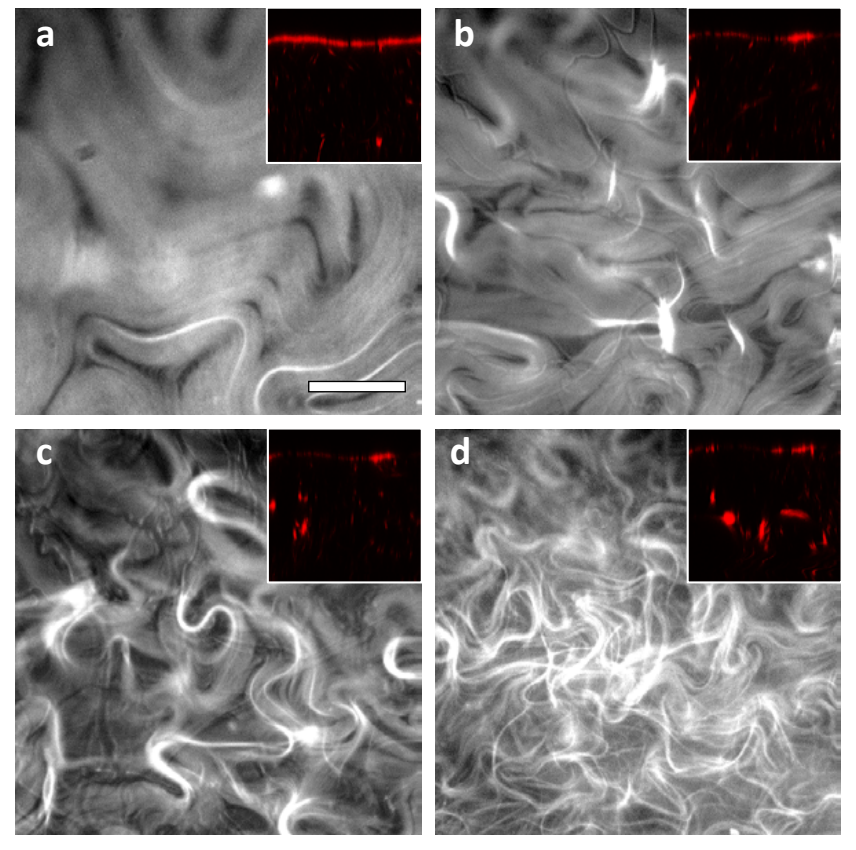

FIG. 5. Fluorescence micrographs showing the structure of the AN in contact with heptadecane as the temperature of the system is continuously lowered from the liquid phase of the oil (a) until the solid phase of the oil (d). In the insets, we observe $125 \mu \mathrm{m}$-wide fluorescence confocal micrographs with cross-sections trough the water/oil interface (oil above water), which appears bright red in (a) due to the presence of the dense AN layer. Fluorescent intensity is proportional to the density of active bundles. The scale bar in (a) is $50 \mu \mathrm{m}$ long.

bulk active gel (Fig. 5d). Using fluorescence confocal microscopy, we confirmed these observations by scanning a cross-section through the water/oil interface. In contact with the liquid oil, the fluorescent active filaments were concentrated at the interface, as corresponds to the AN (Fig. 5a). In contact with the solid oil, the fluorescent material was dispersed in the bulk, as corresponds to the active gel (Fig. 5d). In the process, the system temperature change was lower than $5^{\circ} \mathrm{C}$, to ensure that the properties of the active liquid were not changed significantly by this quench. This process was reversible, and the AN was rapidly reformed upon melting of heptadecane. The reason for this behavior of the AN is so far not understood. In our experiments with silicone oils, we observed the AN to form in contact with oils of extremely high viscosities, without any signature of a threshold viscosity value. We are currently performing studies to explore the role of the yield stress of the contacting isotropic fluid as the relevant rheological parameter for the formation of the AN.

In view of these results, we opted to introduce anisotropy in the interfacial shear stress using a thermotropic liquid crystal instead of an isotropic oil. Liquid crystals are fascinating fluids that feature partial ordering, either orientational or positional, which confers 


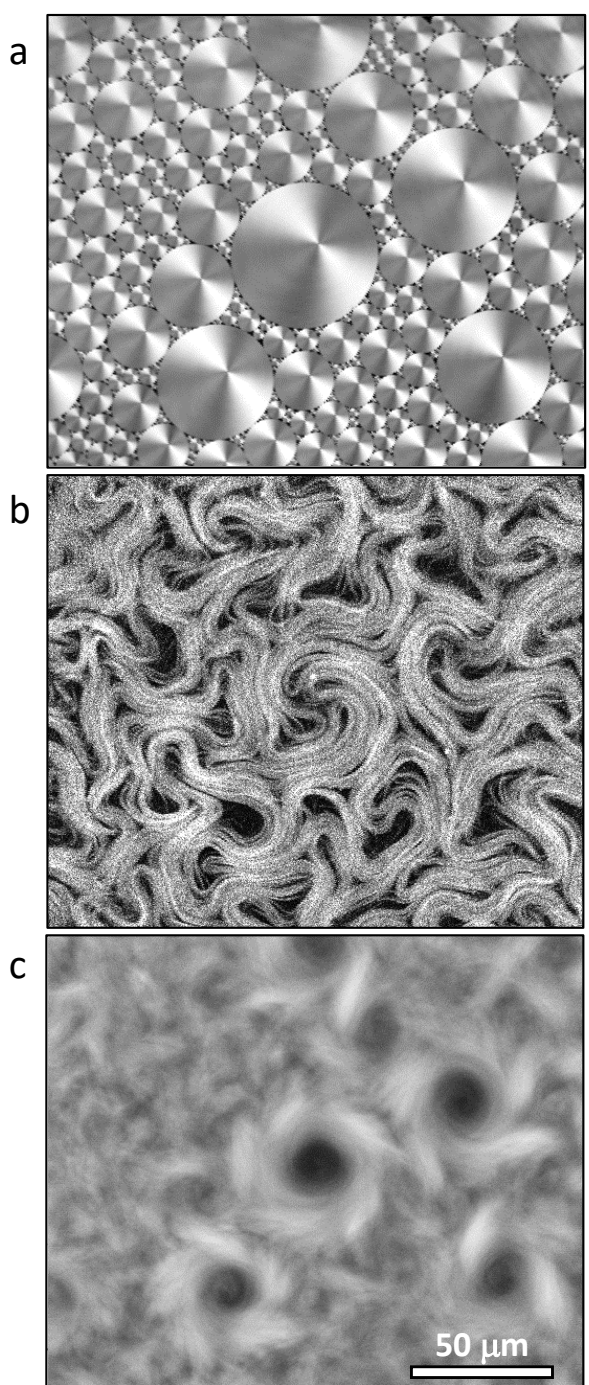

FIG. 6. (a) Confocal reflection micrograph of the $\mathrm{SmA} /$ water interface revealing the spontaneous tiling of the mesogen interface. (b) Fluorescence confocal micrograph of the AN in the active turbulent regime evolving in contact with the tiled SmA interface. (c) A time average of the evolving AN reveals the formation of circulating swirls of different size and persistence, in registry with the underlying SmA domains.

them anisotropy. In our experiments, we chose to work with 8CB (see its description in Sec. II), which features the lamellar smectic-A phase $(\mathrm{SmA})^{38}$ at a temperature range compatible with the active protein suspension. In this phase, molecules are organized in layers, with the average orientation of the rodlike molecules being locally perpendicular to the layers. Although this material behaves macroscopically as a viscous liquid, with an average viscosity of order $10 \mathrm{~Pa} \mathrm{~s}$ at modest shear rates ${ }^{29}$, its rheology is very different at the submillimeter scale: while mesogen molecules can diffuse freely within a layer, transport across layers is hindered. As a result, a $\mathrm{SmA}$ liquid crystal behaves as a liquid for stresses parallel to the layers, but its response is solidlike for stresses in the
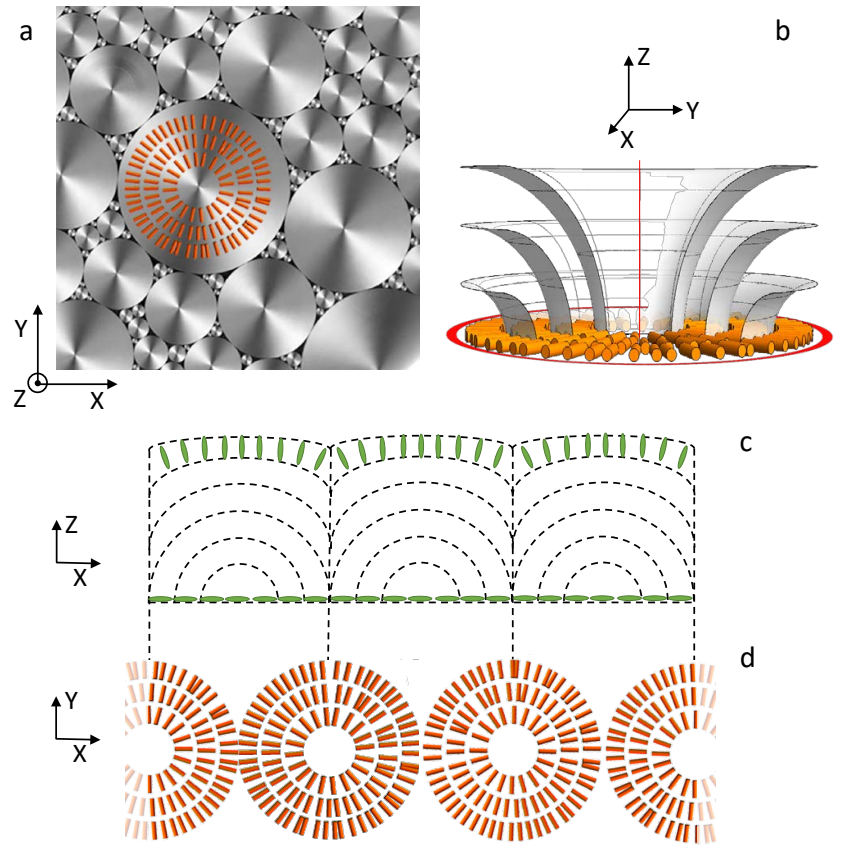

FIG. 7. (a) Confocal reflection micrograph of the lattice of toroidal focal conic domains in the SmA phase. The organization of molecules within one domains is scketed (not to scale). (b) The SmA planes are organized inside the bulk of the SmA phase as truncated tori that have normal intersection at the water/oil interface. (c) Sketch illustrating the corresponding bulk arrangement of the SmA molecules from the planar alignment at the water/oil interface to the homeotropic alignment at the open air interface.

perpendicular direction. We also took advantage of the two main features that make liquid crystals technologically relevant: the possibility to fix the local molecular orientation by means of the boundary conditions at confining interfaces, and the sensitivity of these materials to external electric and magnetic fields, which allows to reversibly control their large-scale molecular orientation.

In Fig. 6, we show a standard open flow cell experiment where the AN formed, not in contact with an isotropic viscous oil, but in contact with $8 \mathrm{CB}$ in the SmA phase. We used fluorescence confocal microscopy for our observations in order to enhance the signal from the AN layer with respect to the active filaments in the bulk aqueous phase. Moreover, our multi-modal instrument enabled to acquire simultaneously the fluorescence signal and the reflection from the polarized excitation laser light. This allowed to capture both the local configuration of the $\mathrm{AN}$ and that of the birefringent SmA liquid crystal. In our experiments, the used surfactant to shield the proteins in the AN from contact with the oil phase also imposed planar anchoring conditions on the SmA molecules, i.e., the mesogen molecules laid parallel to the water interface. In the used open-cell arrangement, the oil was open to the air, where mesogen molecules were oriented perpendicular to the LC/air interface (Fig. 6a). Under 
these hybrid anchoring conditions, the SmA phase organized in a lattice of polydisperse circular domains at the $\mathrm{SmA}$ /water interface. These domains are actually a tiling of the surface, known as Appolonian gasket, and form at the intersection with the interface plane of the complex bulk structures within the SmA phase known as toroidal focal conic domains (TFCDs $)^{38,39}$. In contact with this patterned interface, the AN apparently evolved in the same disordered fashion characteristic of the unconstrained flow regime (Sec. III B). Close inspection, however, revealed that in certain regions the motile $+1 / 2$ defects were organized in rotating swirls (Fig. 6b), where their motion was confined in circular trajectories, different from the random motion in the active turbulent regime $^{28}$.

This new geometry of the AN could be better apprised by looking at the time-average of the active flow (Fig. 6c). It became apparent that the original unconstrained active flow had been spatially structured into a lattice of rotating swirls where the motion of the active filaments was confined. These swirls featured a dark central core, devoid of active material, and were in registry with the underlying lattice of TFCDs. In other words, the circular domains that tile the $\mathrm{SmA}$ /water interface were able to organize the active flow into rotating swirls. By looking at the time-averaged image (Fig. 6c) we noticed that both the size of the swirls and their life time (correlated with how dark the central core is in the time-averaged image) was heterogeneous. Since rotating swirls were the result of the interaction between the $\mathrm{AN}$ and the underlying TFCDs, and we had measured that the latter featured a scale free power law size distribution ${ }^{28}$, we concluded that the exponential distribution of vortex sizes that characterizes the unconstrained turbulent regime was reversibly transformed into a scale-free powerlaw by contact with the patterned interface. Indeed, the process was reversible, as the active turbulent regime was regained if the system was heated above $33.4^{\circ} \mathrm{C}$, where $8 \mathrm{BC}$ transits into the low anisotropy nematic phase. In a different context, a similar change in the scaling behavior has been reported in nematic liquid crystals under turbulent electrohydrodynamical forcing ${ }^{40}$. Concerning the different lifetime of rotative swirls, we observed that only TFCDs above a size comparable to the intrinsic vortex size in the active turbulent regime were able to effectively capture the active flow. As we discuss below, this is one of the remarkable results of our experiments: the AN responds to surface patterning by redefining the role, rather than the value, of the intrinsic length scale $l_{\alpha}$.

The ability of TFCDs to organize the active flow in rotating swirls can be understood by analyzing how $8 \mathrm{CB}$ molecules self-assemble at the interface (Fig. 7). It is known that the SmA planes are organized in concentric tori that intersect the interface at a normal angle. As a result, $8 \mathrm{CB}$ molecules are organized in concentric circular layers and are oriented radially towards the center of the domain. Consistently with the microrheology of the SmA phase explained above, the interface is structured
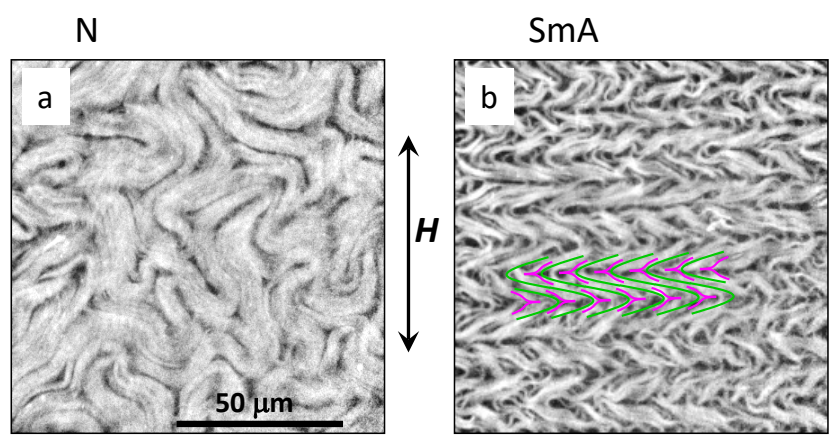

FIG. 8. (a) In contact with $8 \mathrm{CB}$ in the nematic phase, the AN nematic evolves with a geometry indistinguishable from the unconstrained active turbulence, even in the presence of a $0.4 \mathrm{~T}$ magnetic field. (b) Upon a temperature quench into the SmA of $8 \mathrm{CB}$, the turbulent flow is regularized into antiparallel flowing lanes of defects. Images are fluorescence micrographs. The contour of some of the $+1 / 2$ (green) nd $-1 / 2$ (purple) defects are traced in (b).

in concentric circular easy flow directions, while radial flows will be severely hindered. When the AN couples with such micropatterning, the extensile bundles evolve preferentially along the circular easy-flow direction, thus resulting in the observed rotating swirls.

Although this patterning allows to reversibly modify the geometry of active turbulence, the distribution of swirls is set by the random distribution of underlying TFCDs, without any true control capabilities. In the next section, we show that we achieved this control by exploiting the coupling between the thermotropic liquid crystal and a magnetic field.

\section{Forcing with a magnetic field}

The liquid crystal $8 \mathrm{CB}$ features positive diamagnetic anisotropy, which means that, in the presence of a magnetic field, the mesogen molecules will experience a torque that aligns them with the field. Under confinement conditions, where boundaries and interfaces impose a certain anchoring on the molecules, magnetic fields of a few $\mathrm{kG}$ are typically required. In our experiments, we applied a magnetic field of about $4 \mathrm{kG}$ exerted by a custommade Hallbach array of permanent magnets ${ }^{29}$. Using the open flow-cell arrangement described above, we studied the system under the homogeneous magnetic field. The SmA phase, however, was too rigid to respond to this modest magnetic field. Instead, we resorted to a protocol in which we prepared the AN/LC interface with $8 \mathrm{CB}$ in the nematic phase. Although the mesogen molecules align with the magnetic field, the viscous anisotropy in this phase, about a factor of two between flow parallel or perpendicular to the molecules, was too low to have any measurable impact on the geometry of the active turbulent regime (Fig. 8a). We then quenched the temperature below the $\mathrm{SmA}-\mathrm{N}$ phase transition (in practice, 
this meant changing the temperature from $34^{\circ} \mathrm{C}$ down to $33^{\circ} \mathrm{C}$ ). This protocol resulted in a dramatic change in the AN geometry: the random motion of defects typical of active turbulence was regularized into a laminar-like flow, in which defects were organized in antiparallel lanes oriented and moving perpendicularly to the imposed magnetic field (Fig. 8b). Between the lanes, which contained the core of the AN defects, bright stripes contained the active filaments.

This process was reversible, following a similar protocol: the system was heated until the SmA phase melted into the $\mathrm{N}$ phase, thus recovering the active turbulent regime of the $\mathrm{AN}$. At this point, one could change the orientation of the magnetic field and subsequently quench back into the SmA phase, resulting in the reconfiguration of the AN in antiparallel flow lanes aligned perpendicular to the new orientation of the magnetic field. In fact, once $8 \mathrm{CB}$ was in the SmA phase, the magnetic field could be removed, since the configuration of the mesogen could only be altered by melting into the nematic phase.

The physical origin of this indirect coupling between the AN and the magnetic field can be understood by analyzing the structure of the underlying SmA phase that results from a temperature quench in the presence of the magnetic field (Fig. 9). Polarizing microscopy images revealed that $8 \mathrm{CB}$ molecules were oriented, on average, parallel to the imposed magnetic field. The SmA planes, which were perpendicular to the mesogen molecules and to the interface (as explained above) were thus stacked along the magnetic field (Fig. 9). As a result, the easyflow direction was perpendicular to the magnetic field, leading to the observed flow behavior of the AN that coupled to the anisotropic interface. This type of stacking, in which the SmA molecules are organized like books in a bookshelf, is known to propagate into the bulk as parabolic focal conic domains ${ }^{38}$, and can only be modified by first transiting the mesogen to the nematic phase.

\section{E. Intrinsic length and time scale}

In the previous sections we have shown that the geometry of the AN can be reversibly modified by the hydrodynamic coupling with an anisotropic soft interface. In the reported experiments, spatial rearrangement of the active material put into evidence an intrinsic length scale that could be directly and unambiguously probed. In the case of flow over self-assembled SmA interfaces featuring a scale-free distribution of circular domain sizes, the intrinsic length scale emerges as a cutoff size determining the ability of soft domains to capture the active flow. We quantified this trapping ability by measuring the total rotation angle, $\Delta \alpha$, of trapped $+1 / 2$ defects before they escape from the swirl organized by a given TFCD (Fig. 10a). By dividing $\Delta \alpha$ over $2 \pi$, we obtained the number of turns a given defect has performed while being trapped over a particular domain. For the standard experimental conditions of the $\mathrm{AN}$, domains with a di-

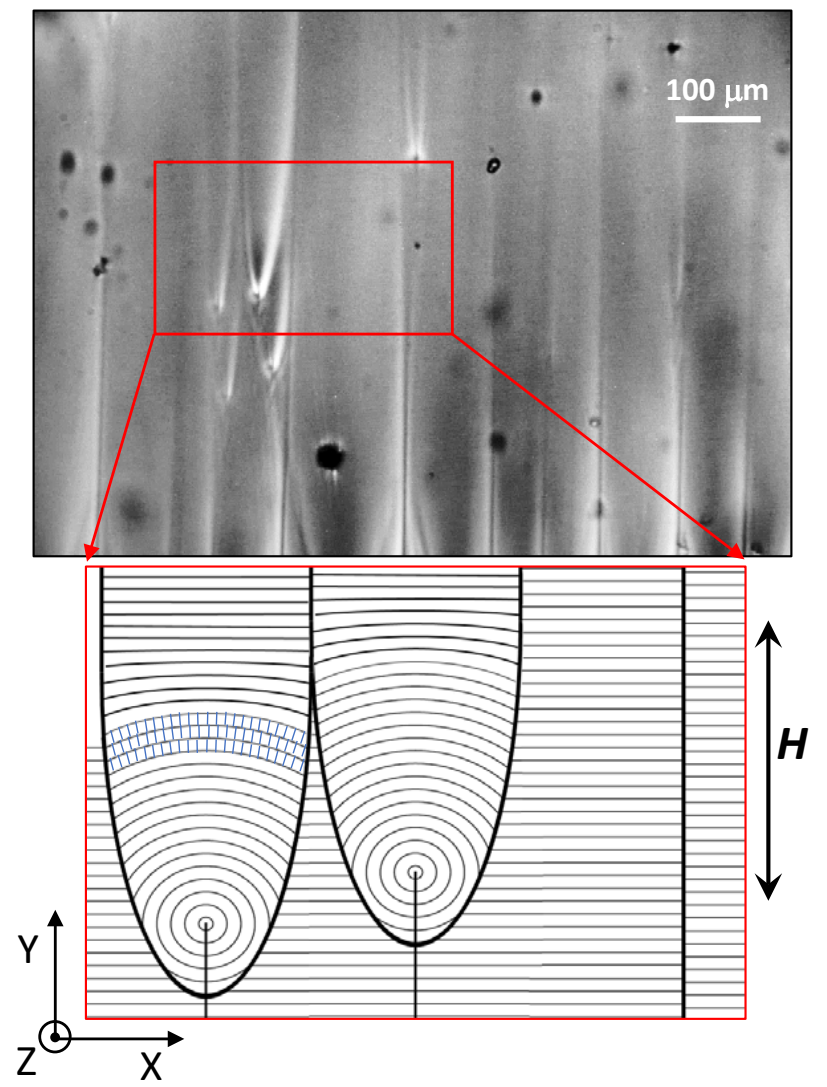

FIG. 9. Polarizing micrograph of the SmA interface aligned by a $0.4 \mathrm{~T}$ magnetic field. The sketch illustrates the bookshelf-like arrangement of SmA planes that intersect perpendicularly to the water interface (XY plane). Thick lines represent straight or parabolic dislocations that are part of the parabolic focal conic domains under this configuration.

ameter less than about $20 \mu \mathrm{m}$ were only able, at most, to scatter the defect trajectories. Conversely, domains with diameter larger that $80 \mu \mathrm{m}$ forced traveling defects to perform more than two full rotations before they were able to escape (Fig. 10b). The fact that the same active length scale that determined the size of vortices and the average spacing between defects in the active turbulent regime also established the threshold domain size for flow capture in the constrained AN can be understood using concepts of defect topology. Each defect has an associated topological charge, defined as the total rotation of the orientational field that features the defect along a circuit that surrounds the defect core. In the case of the $\mathrm{AN}$, the filament orientation performs half a rotation around the defect core (in the same sense as the circuit for $+1 / 2$ defects, and in the opposite sense for $-1 / 2$ defects). However, in a rotating swirl, the active filaments organize a total topological charge +1 , i.e. a full rotation around the circuit. This total charge will be the arithmetic sum of the charges of all the individual defects within the rotating swirl. Since the AN can only form semi-integer defects, there must be, at least, two $+1 / 2$ defects within 
a
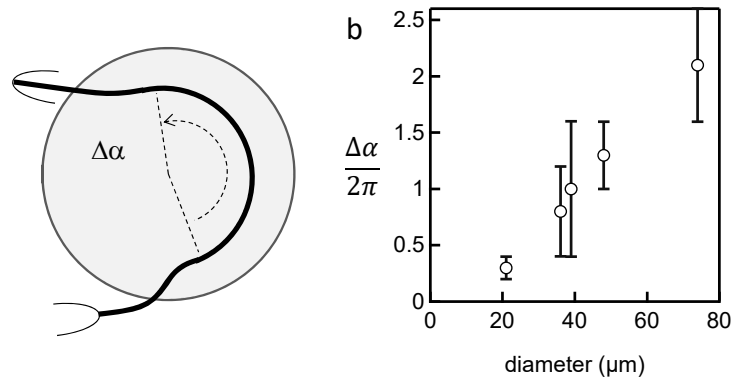

C

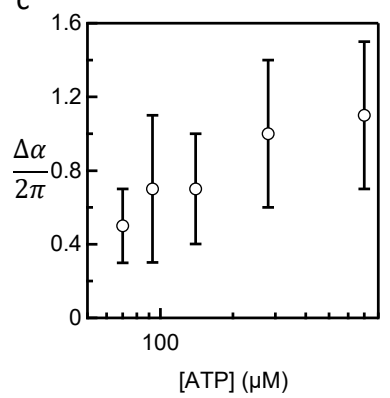

d

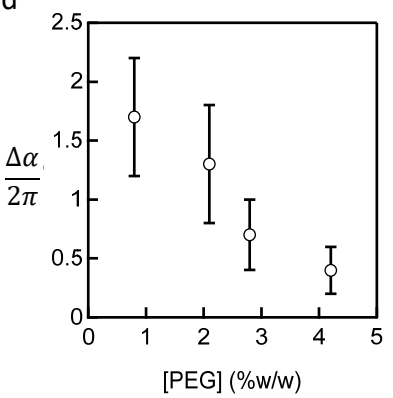

FIG. 10. (a) Sketch illustrating the definition of $\Delta \alpha$, the total angle that a moving $+1 / 2$ defect travels while trapped by the circular domain before moving away. (b) Trapping capability of TFCDs as a function of the domain diameter. Experimental conditions of the $\mathrm{AN}$ are $[\mathrm{ATP}]=700 \mu \mathrm{M}$, $[\mathrm{PEG}]=0.8 \% \mathrm{w} / \mathrm{w}$. (c) Trapping capability of a TFCD of diameter $60 \mu \mathrm{m}$ with an $\mathrm{AN}$ with $[\mathrm{PEG}]=1.6 \% \mathrm{w} / \mathrm{w}$ and different [ATP]. (d) Trapping capability of a TFCD of diameter $60 \mu \mathrm{m}$ with an $\mathrm{AN}$ with $[\mathrm{ATP}]=1.4 \mathrm{mM}$ and different depletant concentration. Error bars are the standard deviation for the measurements.

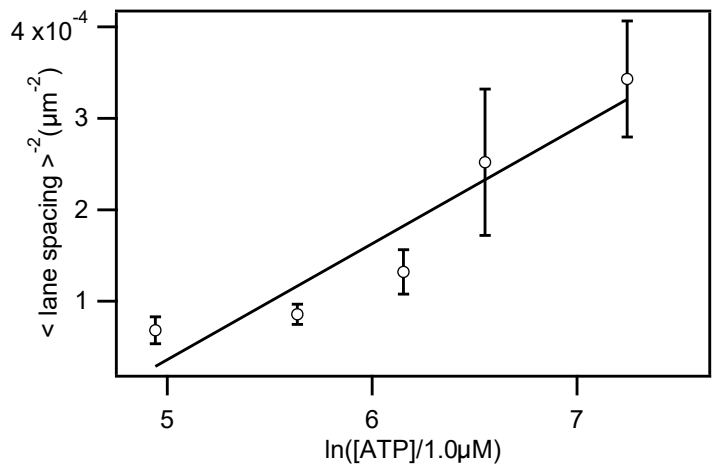

FIG. 11. Lane spacing of the AN in contact with the SmA aligned by a magnetic field. The plot illustrates the scaling with the chemical potential of ATP. The line is a guide for the eye through the data. In these experiments $[\mathrm{PEG}]=$ $0.8 \% \mathrm{w} / \mathrm{w}$.

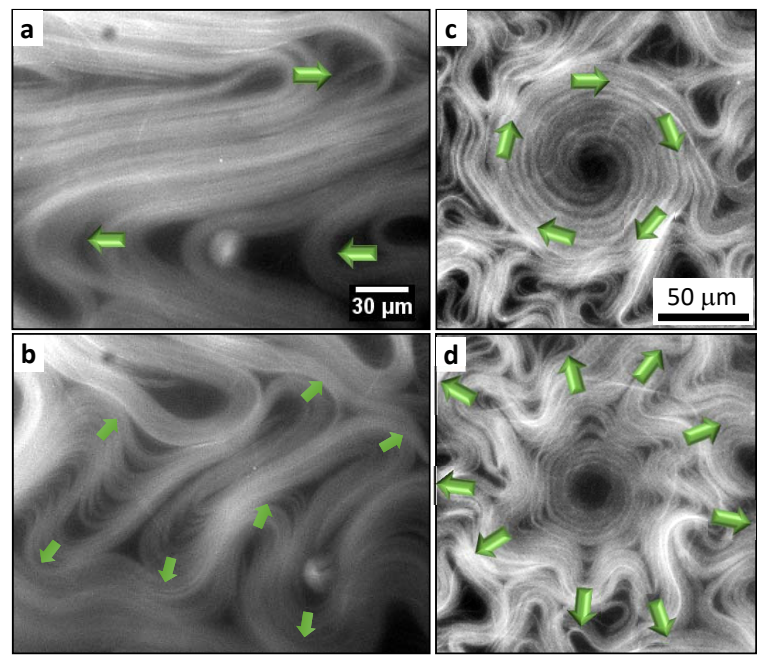

FIG. 12. (a)-(b) Periodic instabilities of the AN aligned by a magnetic field under the same conditions of Fig. 8. The aligned active filament bundles between two antiparallel moving lanes develop periodic bursts of transversal flow. (c)-(d) Periodic instabilities of the AN trapped by a TFCD. Filament bundles are circularly aligned by the rotating swirl (Fig. 6), developing periodic bursts of radial flow. Arrows indicate the direction of the moving $+1 / 2$ defects, which determine the local active flow.

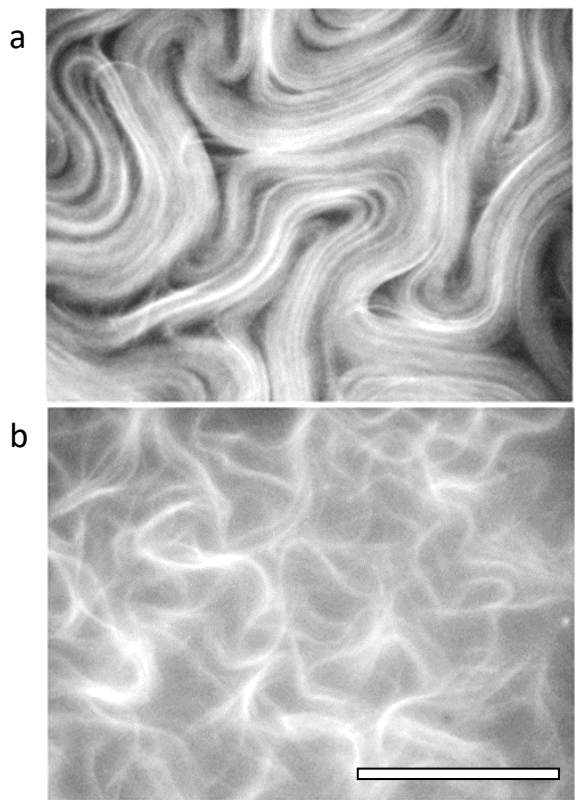

FIG. 13. (a) Fluroescence micrograph with the AN formed at the water/isotropic oil interface using PNIPAM as depleting agent. $T=17.0^{\circ} \mathrm{C}$. (b) After a temperature quench up to $T=20.0^{\circ} \mathrm{C}$, the depletion is weaker, and the AN dissolves. The scale bar in (b) is $100 \mu \mathrm{m}$ long. 
the swirl. This, of course, is the simplest situation: the defect combination can be much more complex, including a balanced number of $+1 / 2$ and $-1 / 2$ defects on condition that their sum equals +1 . While many defects coexist in large swirls, smaller swirls feature a single pair of $+1 / 2 \operatorname{defects}^{28}$. This is, in fact, what determines the smallest swirl size and, therefore, what sets the threshold size for TFCDs to be able to trap the AN flow. Since the minimum separation between defects is set by the active length scale $l_{\alpha}=\sqrt{K / \alpha^{22}}$, and a minimum of two $+1 / 2$ defects is required to organize a rotating swirl, the threshold TFCD size should be proportional to $l_{\alpha}^{2}$. Indeed, we tested this result by using the scaling $l_{\alpha}^{2} \sim \alpha^{-1}$, which predicts that, for a given TFCD size, its trapping ability should increase if we increase the AN activity. Although there is some debate concerning the relation between $\alpha$ and $[A T P]$, we found consistent results when we associated $\alpha$ to the chemical potential of $\mathrm{ATP}^{28,29,34}$, i.e. $\alpha \sim \ln [A T P]$, as seen in Fig. 10c.

Another available control parameter is the concentration of the depleting agent, [PEG], although it is not obvious how $l_{\alpha}$ should depend on this parameter. The simplest assumption is to suppose that only the bending rigidity of the $\mathrm{AN}, K$, is affected by [PEG], presumably leading to an increase in $K$ for higher depletion. We tested such assumption by analyzing the trapping ability of TFCDs for ANs prepared with the same activity but different $[\mathrm{PEG}]$, and found that, indeed, the threshold TFCD size increased with $[\mathrm{PEG}]^{28}$ (Fig. 10d). Nevertheless, this scaling should be further tested, in particular in the context of the length scales put into evidence by the primordial instability (Fig. 2).

In the case of forcing with a magnetic field, the aligned AN develops an obvious spatial modulation, as the flow is organized in antiparallel flow lanes. The spacing between these lanes should be proportional to $l_{\alpha}$, and this led us to analyze this modulation as a function of the activity ${ }^{29}$, showing that the predicted scaling is consistent with our data in the available range of [ATP], again associating the activity to the chemical potential of ATP (Fig. 11).

In the reported constrainment conditions, either with or without an external magnetic field, there were regions where the active bundles were forced to be in a parallel arrangement: in the bright corona surrounding the central dark region in the circulating swirls (Fig. 6) and in the bright stripes between adjacent dark lanes in the magnetic field-aligned AN (Fig. 8). Given the extensile nature of the active bundles, such configurations are unstable with respect to bending of the aligned bundles. As a result, periodic episodes of defect unbinding and motion perpendicularly to the easy flow directions were observed ${ }^{28,29}$ (Fig. 12). In spite of the different geometry in the circularly aligned or in the rectilinearly aligned case, these episodes resulted in clearly measurable velocity oscillations with a well-defined periodicity, which allowed to define an intrinsic time scale for the constrained AN in all cases. Although it was found that this time scale had consistent scaling behavior with the material control parameters, namely, ATP and PEG concentrations $^{28,29}$, it is unclear how to relate it with $l_{\alpha}$ and with the average active flow velocity, which also depends on the average shear viscosity of the oil phase ${ }^{29}$.

\section{CONCLUSION AND OUTLOOK}

The described kinesin/tubulin active nematic is a fascinating active soft material, where the methods and techniques of Soft Matter Physics can be applied to characterize far from equilibrium patterns and dynamic behavior. Our studies took advantage of a crucial result: the strong hydrodynamic coupling between the active nematic film and the bulk fluids on both sides of the interface (the aqueous phase where the protein solution resides and an inert oil at whose interface the AN forms). We have shown that, when the oil is a thermotropic liquid crystal in the SmA phase, the active liquid readily organizes following the local easy flow directions, always exhibiting the same intrinsic active length scale, whose role is defined by the applied perturbations. By patterning the local rheology at the interface, we show that the geometry of the active turbulent regime can be regularized by the interfacial structure. In the case where we forced the active nematic to flow on a lattice of circular domains, the exponential distribution of vortex sizes that characterized the active turbulent regime was regularized into a scale-free power law distribution of rotating swirls. In this case, the intrinsic length scale defined the smallest swirl compatible with the active material topology. In the case where the bulk mesogen was aligned with a magnetic field, the turbulent active flow was organized in antiparallel flowing lanes. In all cases, intrinsic length and time scales were extracted, and their scaling value with material control parameters, namely, the concentration of ATP and that of the depleting agent PEG, were assessed.

The use of an external magnetic field has afforded some degree of reversible and reconfigurable control on the geometry of the active nematic. Nevertheless, this control is external to the material, possible thanks to the hydrodynamic coupling at the interface. We are currently considering a direct control strategy of the state of the active nematic by using as depletant the well-known thermosensitive polymer PNIPAM (Poly(N-isopropylacrylamide)), whose radius of giration in the aqueous solution can be changed with temperature within a biocompatible range, thus allowing to alternate between two depletion regimes. This could allow to switch between the active nematic and the bulk active gel states (Fig. 13), thus providing with a handle to set the initial condition even in situations where the active material is in a strictly confined geometry, such as in spherical encapsulation.

In spite of the advances in the control and characterization of the active nematic reported in the present work, many open questions remain. For instance, it is still unclear how the concentration of PEG modifies the bending 
rigidity, and its scaling within the active length scale. It is also unresolved how the intrinsic time scale that we put into evidence in our experiments scales with the different control parameters. These issues are currently being addressed in the context of the primordial instability (Fig. 2) where the well-defined initial condition should allow to carefully isolate the effect of the different material parameters.

\section{ACKNOWLEDGMENTS}

We are indebted to Z. Dogic and S. DeCamp (Brandeis University), and the Brandeis University MRSEC Biosynthesis facility for their assistance in the preparation of the active gel. We thank B. Hishamunda (Brandeis University), and M. Pons, A. LeRoux, and G. Iruela (Universitat de Barcelona) for their assistance in the expression of motor proteins. We thank R. Casas and G. Valiente (Bluestar Silicones) for providing silicone oil samples. Funding has been provided by MINECO (projects FIS 2013-41144P and FIS2016-78507-C2-1-P AEI/FEDER-EU). P.G. acknowledges funding from Generalitat de Catalunya through a FI-DGR PhD Fellowship. J. H. acknowledges funding from a Marie-Curie PhD fellowship in the NANOTRANS European project. B.M.P acknoledges partial funding by Maria de Maeztu Programme for Units of Excellence in R\&D (MDM-20140377). Brandeis University MRSEC Biosynthesis facility is supported by NSF MRSEC DMR-1420382.

${ }^{1}$ S. Ramaswamy, Annual Review of Condensed Matter Physics 1, 323 (2010).

${ }^{2}$ M. C. Marchetti, J. F. Joanny, S. Ramaswamy, T. B. Liverpool, J. Prost, M. Rao, and R. A. Simha, Reviews of Modern Physics 85, 1143 (2013).

${ }^{3}$ S. Kohler, V. Schaller, and A. R. Bausch, Nat Mater 10, 462 (2011).

${ }^{4}$ Y. Sumino, K. H. Nagai, Y. Shitaka, D. Tanaka, K. Yoshikawa, H. Chate, and K. Oiwa, Nature 483, 448 (2012).

${ }^{5}$ T. Sanchez, D. T. Chen, S. J. DeCamp, M. Heymann, and Z. Dogic, Nature 491, 431 (2012).

${ }^{6}$ S. R. Nagel, Reviews of Modern Physics 89, 025002 (2017).

${ }^{7}$ E. Tjhung, D. Marenduzzo, and M. E. Cates, Proc Natl Acad Sci U S A 109, 12381 (2012).

${ }^{8}$ M. Ravnik and J. M. Yeomans, Phys Rev Lett 110, 026001 (2013).

${ }^{9}$ L. Giomi and A. DeSimone, Physical Review Letters 112, 147802 (2014).

${ }^{10}$ F. C. Keber, E. Loiseau, T. Sanchez, S. J. DeCamp, L. Giomi, M. J. Bowick, M. C. Marchetti, Z. Dogic, and A. R. Bausch, Science 345, 1135 (2014).

${ }^{11}$ J. Alvarado, B. M. Mulder, and G. H. Koenderink, Soft Matter 10, 2354 (2014).
${ }^{12}$ K. T. Wu, J. B. Hishamunda, D. T. Chen, S. J. DeCamp, Y. W. Chang, A. Fernandez-Nieves, S. Fraden, and Z. Dogic, Science 355, eaal1979 (2017).

${ }^{13}$ D. E. Discher, P. Janmey, and Y. L. Wang, Science 310, 1139 (2005).

${ }^{14}$ R. Sunyer, V. Conte, J. Escribano, A. Elosegui-Artola, A. Labernadie, L. Valon, D. Navajas, J. M. Garcia-Aznar, J. J. Munoz, P. Roca-Cusachs, and X. Trepat, Science 353, 1157 (2016).

${ }^{15}$ B. Alberts, Molecular biology of the cell, 4th ed. (Garland Science, New York, 2002).

${ }^{16}$ D. E. Jaalouk and J. Lammerding, Nat Rev Mol Cell Biol 10, 63 (2009).

${ }^{17}$ F. Carbone, H. Yoshida, S. Suzuki, A. Fujii, G. Strangi, C. Versace, and M. Ozaki, EPL (Europhysics Letters) 89 (2010).

${ }^{18}$ E. Lushi, H. Wioland, and R. E. Goldstein, Proc Natl Acad Sci U S A 111, 9733 (2014).

${ }^{19}$ C. Peng, T. Turiv, Y. Guo, Q. H. Wei, and O. D. Lavrentovich, Science 354, 882 (2016).

${ }^{20}$ H. H. Wensink, J. Dunkel, S. Heidenreich, K. Drescher, R. E. Goldstein, H. Lowen, and J. M. Yeomans, Proc Natl Acad Sci U S A 109, 14308 (2012).

${ }^{21}$ S. P. Thampi, R. Golestanian, and J. M. Yeomans, Philos Trans A Math Phys Eng Sci 372, 20130366 (2014).

${ }^{22}$ L. Giomi, Physical Review X 5, 031003 (2015).

${ }^{23}$ V. Bratanov, F. Jenko, and E. Frey, Proc Natl Acad Sci U S A 112, 15048 (2015).

${ }^{24}$ S. P. Thampi and J. M. Yeomans, The European Physical Journal Special Topics 225, 651 (2016).

${ }^{25}$ S. P. Thampi, A. Doostmohammadi, T. N. Shendruk, R. Golestanian, and J. M. Yeomans, Sci Adv 2, e1501854 (2016).

${ }^{26}$ A. Doostmohammadi, T. N. Shendruk, K. Thijssen, and J. M. Yeomans, Nat Commun 8, 15326 (2017).

${ }^{27}$ P. Guillamat, J. Ignes-Mullol, S. Shankar, M. C. Marchetti, and F. Sagues, Physical Review E 94, 060602 (2016).

${ }^{28}$ P. Guillamat, Ignes-Mullol, and F. Sagues, Nat Commun 8, 564 (2017).

${ }^{29}$ P. Guillamat, J. Ignes-Mullol, and F. Sagues, Proc Natl Acad Sci U S A 113, 5498 (2016).

${ }^{30}$ K. Kruse, J. F. Joanny, F. Julicher, J. Prost, and K. Sekimoto, Eur Phys J E Soft Matter 16, 5 (2005).

${ }^{31}$ F. Julicher, K. Kruse, J. Prost, and J. F. Joanny, Physics Reports 449, 3 (2007).

${ }^{32}$ J. Prost, F. Jlicher, and J. F. Joanny, Nature Physics 11, 111 (2015).

${ }^{33}$ X. Q. Shi and Y. Q. Ma, Nat Commun 4, 3013 (2013).

${ }^{34}$ S. P. Thampi, R. Golestanian, and J. M. Yeomans, Phys Rev Lett 111, 118101 (2013).

${ }^{35}$ C. A. Weber, C. Bock, and E. Frey, Phys Rev Lett 112, 168301 (2014).

${ }^{36}$ J. Dunkel, S. Heidenreich, K. Drescher, H. H. Wensink, M. Bar, and R. E. Goldstein, Phys Rev Lett 110, 228102 (2013).

${ }^{37}$ R. Benzi, S. Patarnello, and P. Santangelo, Journal of Physics A: Mathematical and General 21, 1221 (1988).

${ }^{38} \mathrm{P}$. Oswald and P. Pieranski, Smectic and columnar liquid crystals : concepts and physical properties illustrated by experiments, The liquid crystal book series (Taylor and Francis, Boca Raton, FL, 2006).

${ }^{39}$ C. Blanc and M. Kleman, Physical Review E 62, 6739 (2000).

${ }^{40}$ F. Carbone, L. Sorriso-Valvo, C. Versace, G. Strangi, and R. Bartolino, Phys Rev Lett 106, 114502 (2011). 